\title{
Prevalence And Distribution Of Dental Caries Experience According To GV Black Classification For Patient Attending To Dental School
}

Talabani RM ${ }^{1}$, Al-Zahawi A², Ibrahim RO

\begin{abstract}
Objective: The aim of our study was to evaluate the incidence of dental caries in relation to gender. GV Black classification was used.

Method: A sample of 1000 adult patient, 498 male and 502 female, were selected at random from patient attending to dental clinics of Conservative department at School of Dentistry, University of Sulaimani.

Result: According to GV Black classification of dental caries (7885) carious lesions were detected on various surfaces, class I buccal surface 387 (4.9\%), class I palatal surface 465 (5.8 \%), class I occlusal surface 4836 (61.3\%), class II mesio-occlusal (MO) 499 (6.3\%), class II disto-occlusal (DO) 594 (7.5\%), class II mesio-occlusal-distal (MOD) 215 (2.7\%), class III mesial 331 (4.1\%), class III distal 289 (3.6\%), class IV 95 (1.2\%), class V 174 were (2.2\%).
\end{abstract}

Conclusion: The result of this study showed that Class I occlusal surface caries was more prevalent among other types of carious lesion according to GV Black classification.

Keywords: Dentition, GV Black caries classification, Diagnosis

${ }^{1}$ Lecturer

Conservative Department,

School of Dentistry, Faculty of Medical Sciences, University of Sulaimani

${ }^{2}$ Lecturer

Conservative department

School of Dentistry, Faculty of Medical Sciences, University of Sulaimani

${ }^{3}$ Assisstant lecturer

Conservative Department

School of Dentistry, Faculty of Medical Sciences,

University of Sulaimani

\section{Contact Author}

\section{Dr. Ranjdar Mahmood Talabani ranjdartalabani@yahoo.com ranjdar.osman@univsul.com}

J Oral Health Comm Dent 2015;9(2)60-63

\section{INTRODUCTION}

7 he majority of industrialized countries have experienced a significant reduction in the prevalence of dental caries due to the continuous improvement of living conditions, improved self-care practices, effective use of fluorides, adoption of healthy lifestyles and implementation of preventive oral care programs while in developing countries increasing level of dental caries and treatment need has been observed (1).

Dental caries is the most common chronic disease worldwide and affects approximately $90 \%$ of adults in the
US according to the United States National Institutes of Health Consensus Development Panel [2001]; more than two-thirds of American adults aged 35 to 44 years have lost at least one tooth due to dental caries(2).

Published report revealed a wide diversity in the prevalence of dental caries among adult and geriatric populations, this was attributed to environmental and host factors (3).

Dental caries is a multifactorial disease; several factors play a role in the initiation and progression of the lesion including environmental factors, host, and behavioral factors (2). 
The distribution of caries has changed in the last century and exclusively recent data indicate that about 90 percent of carious lesions occur in the pits and fissures of permanent posterior teeth and that molar tooth are most susceptible to caries(4).

Background knowledge on dental caries including prevalence, extension and severity provides a baseline data for the eminence essence of caries preventive programs and treatment need in populations which pose a greater burden as it financially and negatively affects the quality of life of an individual (5).

In the recent years the global distribution of dental caries presents a varied picture, most of the countries with low caries prevalence are experiencing an unprecedented increase in caries prevalence and severity of dental caries hence the present study was aimed at determining the prevalence of dental caries, effect of the gender and the positioning of the teeth and their surfaces in the oral cavity on the prevalence of dental caries.

\section{METHOD}

Patients were selected from the outpatient departments of Conservative Dentistry/ School of Dentistry, University of Sulaimani. Specialized form was presented at conservative department for clinical operative purpose including (DMF index) used to record the incidence of caries distribution according to GV Black classification among patients.

A diagnostic criteria for clinical examination was followed wherein, caries was detected according to WHO criteria that "clinically the caries was recorded as present when a lesion in a pit or fissure or on a smooth surface had a detectable softened floor, undermined enamel or softened wall. On Proximal surface it had to be certain that the explorer had entered the lesion or taking bitewing radiograph for any doubt existed caries"(5).
Black in 1908 classified carious lesion by location as follow:

- Class I: These are including occlusal surface, buccal and lingual pits of posterior teeth and lingual surface of anterior teeth.

- Class II: These are involving proximal surface of posterior teeth.

- Class III: These are involving proximal surface of anterior teeth that do not include the incisal edge.

- Class IV: These are involving the proximal surface of anterior teeth that include the incisal edge

- Class V: These are involving the gingival third of the facial or lingual surface of all teeth (6).

Carious lesions that were found on the incisal edge or cusp tips were excluded from the present study as it's not recommended by GV Black.

\begin{tabular}{|c|c|c|c|}
\hline Class & Male & Female & Total \\
\hline CL 1 buccal & 143 & 244 & 387 \\
\hline CL 1 palatat & 201 & 264 & 465 \\
\hline CL 1 occlussal & 2366 & 2470 & 4836 \\
\hline CL $2 \mathrm{MO}$ & 206 & 293 & 499 \\
\hline CL 2 DO & 294 & 300 & 594 \\
\hline CL 2 MOD & 83 & 132 & 215 \\
\hline CL 3 mesial & 133 & 198 & 331 \\
\hline CL 3 distal & 132 & 157 & 289 \\
\hline CL4 & 51 & 44 & 95 \\
\hline CL 5 & 98 & 76 & 174 \\
\hline Total & 498 & 502 & 1000 \\
\hline
\end{tabular}

\section{RESULT}

Figure 1, demonstrates the distribution

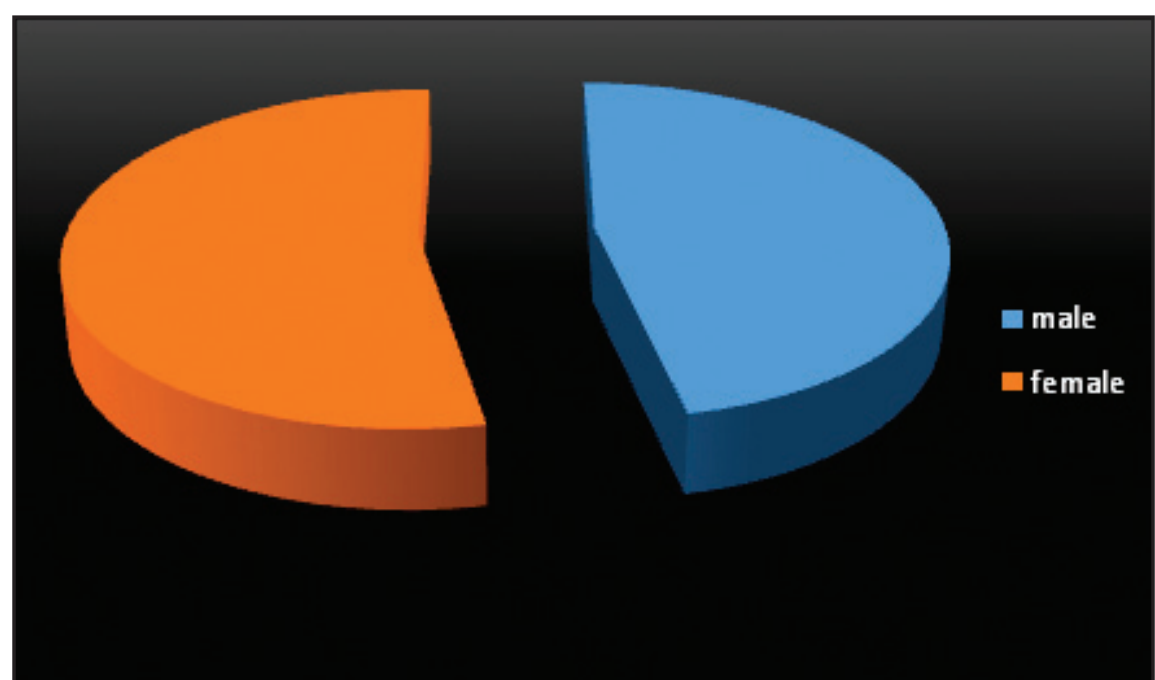

Figure 1: Male and Female Distribution

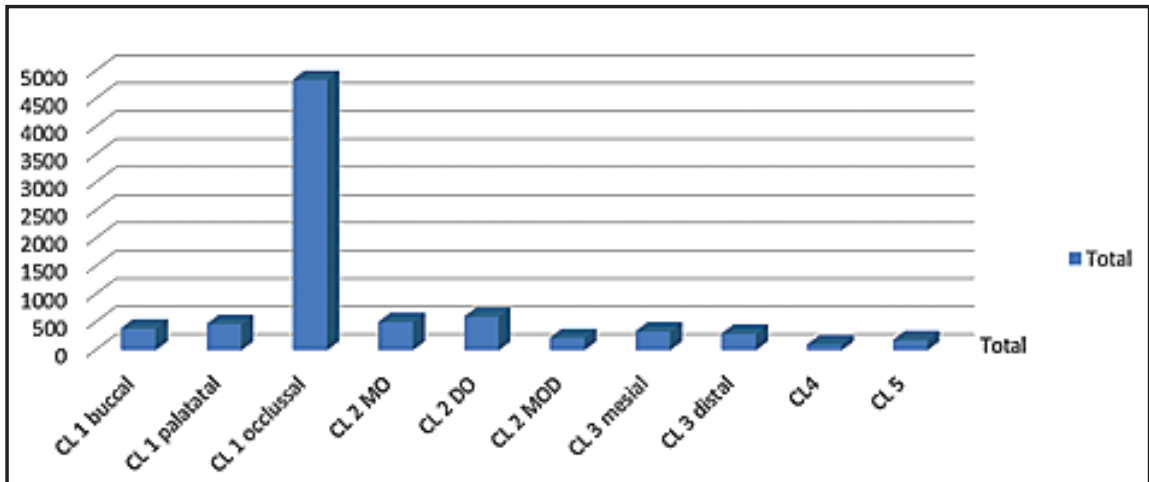

Figure 2: Distribution of dental caries lesion according to GV Black classification 


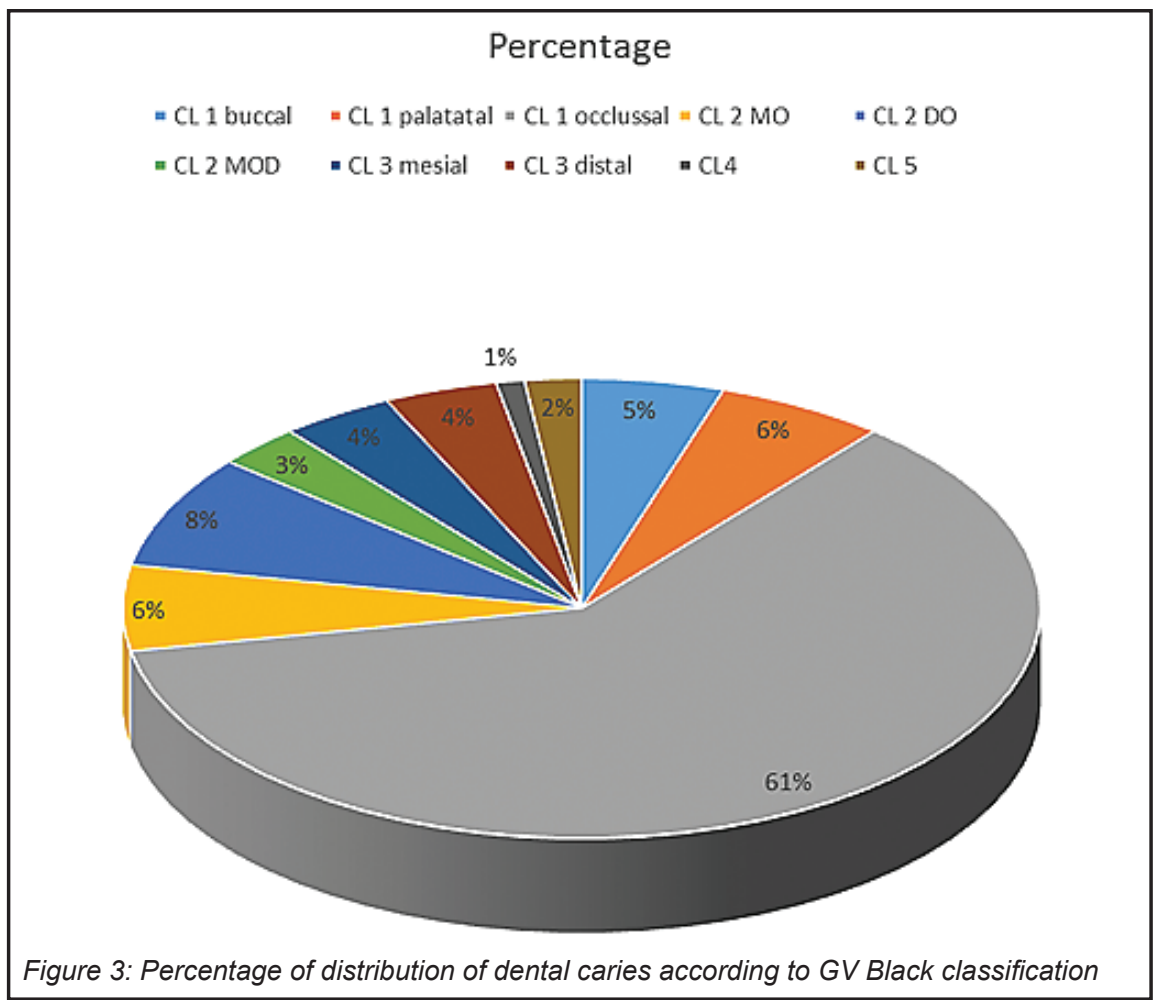

of the study sample which consist of 1000 patient, 498 male and 502 female were recruited for this study.

A total of 7885 carious lesions were

detected as shown in table (1),class I buccal surface $387(4.9 \%)$, class I palatal surface 465 (5.8\%), class I occlusal surface $4836(61.3 \%)$, class II mesio-occlusal (MO) 499 (6.3\%), class

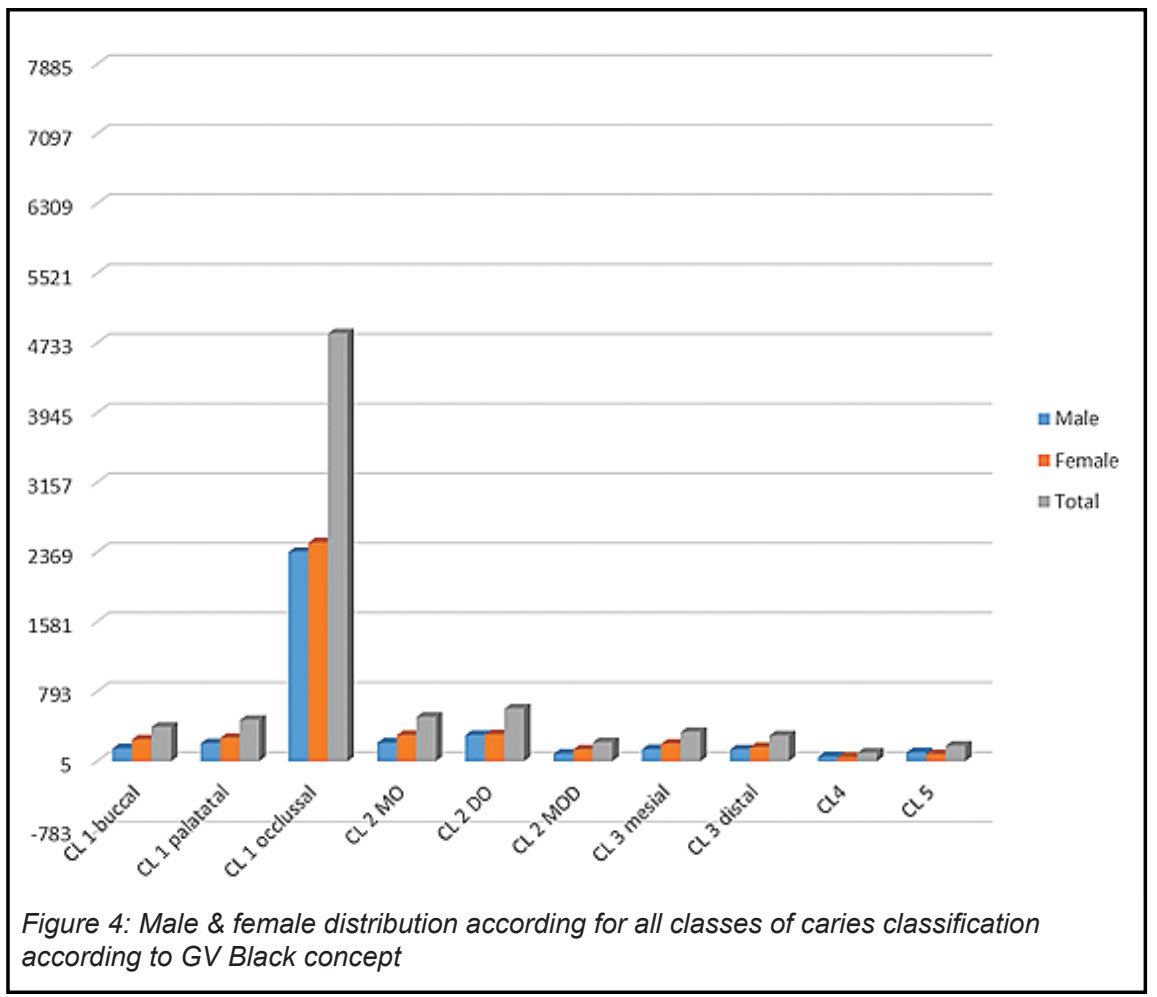

II disto-occlusal (DO) 594 (7.5\%), class II mesio-occlusal-distal (MOD) 215 (2.7 $\%)$, class III mesial 331 (4.1\%), class III distal 289 (3.6\%), class IV 95 (1.2\%), class V 174 were $(2.2 \%)$ as shown in Fig. (2 and 3).

Figure (4) demonstrates surface distribution of caries lesion according to GV Black's classification among male and female, as shown in the figure, percentages of surface lesions for all classes was more in female than male (class I buccal, male $36.9 \%$ and female $63.04 \%$, class I palatal, male $43.2 \%$ and female $56.7 \%$, class I occlusal, male $48.9 \%$ female $51.07 \%$, class II mesioocclusal, male 41.2 and female 58.7 $\%$, class II disto-occlusal, male $49.4 \%$ female $50.5 \%$, class II mesio-occlusaldistal, male $38.6 \%$ and female $61.3 \%$, class III mesial, male $40.1 \%$ and female $59.8 \%$, class III distal, male $45.6 \%$ and female $54.3 \%$ ). Apart of class V lesions, female recorded higher percentages of all classes of dental caries than male. Whereas, distribution of class $\mathrm{V}$ carious lesion among males was more than female (male $56.3 \%$ and female $43.6 \%$ ) and class IV (male $53.6 \%$ and female $43.6 \%)$.

\section{DISCUSSION}

The present study was conducted to evaluate the prevalence and distribution of dental caries among both genders according to GV Black classification of dental caries which was established in 1910.

The incidence of dental caries has been extensively studied over the past 50 years in North America and Europe. Epidemiological studies have been useful in determining the extent for effectiveness of dental treatment. The most common measures of caries are an evaluation of the number of permanent teeth that are decayed, missing, or filled (DMF). Measures of primary teeth are reported as (DMF). This measure is cumulative as it totals the number of restorations and extractions in addition to the number of teeth having 
active caries. DMF scores are not the true equivalent of caries prevalence as they overstate the prevalence of active caries (7).

In this study G.V.Black's classification was used, which is more basic, practical and appealing. This clinical detection criteria of caries was simple and straight forward. Cavitated and non-cavitated lesions are easily identified and classified.

The result of this study showed that class I occlusal surface lesion was very high $(61.3 \%)$ compared with the other classes, this was in accordance with a study done by Muntaha et al, (2013) (7). However, class II, class III, class I palatal (lingual), class I buccal, class II mesio-occlusal-distal, class $\mathrm{V}$ and class IV recorded lesser prevalence of dental caries respectively and this agree with study done by SantiandSubbarao (2012) (8).

In our study higher caries rates among female for all classes except for class IV and class $\mathrm{V}$ which is well documented in dental studies of contemporary human populations and this is in agree- ment with study done by LukacsandLargaespada (2006) (9)and study done by Azam et al, (2011)(10) which stated that recent clinical and experimental caries research confirm the impact of hormonal fluctuations and pregnancy on the quantity and quality of saliva, and thereby on oral ecology, so the physiological mechanisms by which life-history events have a more direct and significant influence on poorer dental health in women than in men is becoming clearer.

\section{CONCLUSION}

The current study recorded highest prevalence of class I lesions compared to other classes of GV Black's classification, whereas class IV and class $\mathrm{V}$ scored the least percentages among the population examined in this study. Furthermore, female found more likely to experience caries than male.

\section{REFERENCES:}

1. Baciu D, Danila I andBalcos. Exploring the association of caries experiencing with social and behavioral factors among schoolchildren from IASI, Romania. Romanian Journal of Oral Rehabilitation 2013;5(3).

2. Shaffer JR, Feingold E, Wang X, Lee M, TCuenco K, Weeks DE, et al. GWAS of dental caries patterns in the permanent dentition. J Dent. Res 2013:92(38).

3. Sonbul H. On caries risk profile and prevention in an adult Saudi population. MSc thesis, Department of Cariology Institute of Odontology at Sahlgrenska Academy University of Gothenburg, Sweden, 2010.

4. Zero DT, Fontana M, Mier EAM, Zandona AF, Ando M, Cabezas CG et al. The biology, prevention, diagnosis and treatment of dental caries Scientific advances in the United States. JADA 2009;140(9 suppl):25-34.

5. Hegda MN, Hegda P, Bhat R. Prevalence of dental caries in anterior teeth in South Canara population- a three year epidemiological study. IJAR 2014;4(2).

6. Nomann NA, Polan MAA, Jan CM, Rashid $\mathrm{F}$ andTaleb A. Amalgam and composite restoration of posterior teeth. Bangladesh J Dent Res Educ 2013;3(1).

7. Ali Shah SH, Muntaha S, Munir MB. Incidence of caries in 6-12 years children visting Punjab dental hospital, Lahore andSardarBegum dental college and hospital, Peshawar. Pak Oral Dental J 2008;28(1).

8. Santi V, Subbarao V. Centuries of Conservative Dentistry. J Interdiscip Dentistry 2012;1(1).

9. Lukacs JR, Largaespada LL. Explaining sex differences in dental caries prevalence: saliva, hormones, and "life-history" etiologies. Am J Hum Biol 2006;18:540-55.

10. Azam S, Khurram MS, Hassan M, Iqbal F, lqbal S. Distrbution of dental caries and its relationship to risk factor. Pak Oral Dental J 2011;31(2). 